\title{
Assessing Mild Cognitive Impairment with Amyloid and Dopamine Terminal Molecular Imaging
}

\author{
Roger L. Albin ${ }^{1-4}$, James F. Burke ${ }^{1,3,5}$, Robert A. Koeppe ${ }^{6}$, Bruno Giordani ${ }^{4,7}$, Sid Gilman ${ }^{1}$, and Kirk A. Frey ${ }^{1,6}$ \\ ${ }^{1}$ Department of Neurology, University of Michigan, Ann Arbor, Michigan; ${ }^{2}$ Geriatrics Research, Education, and Clinical \\ Center, VAAAHS, Ann Arbor, Michigan; ${ }^{3}$ Neurology Service, VAAAHS, Ann Arbor, Michigan; ${ }^{4}$ Michigan Alzheimer Disease \\ Center, Ann Arbor, Michigan; ${ }^{5}$ Robert Wood Johnson Clinical Scholars Program, University of Michigan, Ann Arbor, Michigan; \\ ${ }^{6}$ Department of Radiology, University of Michigan, Ann Arbor, Michigan; and ${ }^{7}$ Department of Psychiatry, University of Michigan, \\ Ann Arbor, Michigan
}

We evaluated PET-based classification of neurodegenerative pathology in mild cognitive impairment $(\mathrm{MCl})$. Methods: Our study was a cross-sectional and prospective evaluation of a cohort of $27 \mathrm{MCl}$ subjects drawn from a university-based cognitive disorders clinic. We compared expert clinical consensus classification of $\mathrm{MCl}$ at entry and possible dementia at follow-up with molecular imaging-based classification using ${ }^{11} \mathrm{C}$-dihydotetrabenazine PET measurement of striatal dopamine terminal integrity and ${ }^{11} \mathrm{C}$-Pittsburgh compound $\mathrm{B}\left({ }^{11} \mathrm{C}-\mathrm{PiB}\right) \mathrm{PET}$ measurement of cerebral amyloid burden. Results: Eleven subjects were initially classified clinically as amnestic $\mathrm{MCl}, 7$ as multidomain $\mathrm{MCl}$, and 9 as nonamnestic $\mathrm{MCl}$. At a mean follow-up of $3 \mathrm{y}$, 18 subjects converted to dementia. PET imaging evidence of significant cerebral amyloid deposition or nigrostriatal denervation was a strong predictor of conversion to dementia. There was only moderate concordance between expert clinical classifications and PET-based classifications of dementia subtypes. Conclusion: Combined PET molecular imaging of cerebral amyloid burden and striatal dopamine terminal integrity may be useful for identifying subjects at high risk for progression to dementia and in defining neurochemically differentiated subsets of $\mathrm{MCl}$ subjects.

Key Words: dementia; Alzheimer disease; Lewy body dementia; frontotemporal dementia

J Nucl Med 2013; 54:887-893

DOI: 10.2967/jnumed.112.112599

$\mathbf{M}$ ild cognitive impairment (MCI) is recognized as a substantial risk factor for subsequent development of dementia (1). There is considerable interest in MCI subjects as suitable target populations for clinical trials aimed at delaying dementia onset. MCI, however, is a heterogeneous entity caused by all major neurodegenerative pathologies and vascular etiologies (1-7). Some MCI subjects do not

\footnotetext{
Received Aug. 9, 2012; revision accepted Dec. 4, 2012.

For correspondence or reprints contact: Roger L. Albin, 5023 BSRB, 109 Zina Pitcher PI., Ann Arbor, MI, 48109-2200.

E-mail: ralbin@umich.edu

Published online Apr. 9, 2013.

COPYRIGHT @ 2013 by the Society of Nuclear Medicine and Molecular Imaging, Inc.
}

have a progressive course, and others may improve over time (8-12). Clinical trials enrolling MCI subjects face the same challenge as trials involving subjects with early dementias: the strong likelihood of enrolling heterogeneous subject populations, plus the problem of enrolling subjects who will not go on to develop dementia. The development of PET ligands identifying characteristic pathologic features of different neurodegenerative dementias offers the possibility of a minimally invasive subclassification of MCI subjects based on well-characterized correlates of pathology $(13,14)$. Because potential treatments to prevent or delay onset of dementia are likely to target specific pathologic processes, the identification of subject groups with specific underlying pathologic features is likely to improve the statistical power of clinical trials.

There are 3 primary causes of neurodegenerative dementias-Lewy body dementia (LBD), Alzheimer disease (AD), and frontotemporal dementias (FTDs). LBD is characterized by substantial loss of nigrostriatal dopaminergic terminals, together with cerebrocortical Lewy bodies and Lewy neurites. AD and a significant percentage of LBD subjects exhibit neuritic plaques composed of fibrillar amyloid precursor protein fragments (A $\beta$ amyloid). FTDs generally lack these features and are characterized by deposition of a variety of protein species including $\tau$-protein and TAR-DNAbinding protein- 43 .

Studies correlating amyloid imaging results with the postmortem assessments of amyloid burden indicate a good correlation between imaging and pathologic measures of amyloid burden (15). Prior postmortem studies evaluating nigrostriatal degeneration in pathologically defined $\mathrm{AD}$ and LBD samples indicated a high specificity for substantial loss of nigrostriatal dopaminergic terminals as a marker for LBD $(16,17)$. These results, and others, indicate that molecular imaging with amyloid and nigrostriatal dopamine terminal ligands may identify characteristic pathologic features of neurodegenerative dementias.

We previously reported PET imaging-based classification of early, mild dementias with amyloid and dopamine terminal molecular imaging. We demonstrated only moderate (Cohen's $\kappa=0.39)$ concordance between molecular imaging-based 
classifications and expert consensus-based clinical classifications (18). To clarify the potential role of this imaging-based approach in the subclassification of MCI and to evaluate the prediction of progression to dementia, we report the results of combined PET imaging with the nigrostriatal dopamine terminal marker ${ }^{11} \mathrm{C}$-dihydotetrabenazine and the $\mathrm{A} \beta$ amyloid marker ${ }^{11} \mathrm{C}$-Pittsburgh compound $\mathrm{B}\left({ }^{11} \mathrm{C}-\mathrm{PiB}\right)$ in a cohort of MCI subjects.

\section{MATERIALS AND METHODS}

\section{Subjects}

Twenty-seven subjects with primary symptoms of cognitive impairment were recruited from the University of Michigan Cognitive Disorders Clinic. This was a convenience sample of subjects meeting criteria for MCI and satisfying the following inclusion and exclusion criteria (18). Included subjects were over the age of 40, had cognitive symptoms for longer than $9 \mathrm{mo}$, and were capable of completing neuropsychologic testing and research neuroimaging. Subjects with a modified Hachinski scale score greater than 4 or meeting NINDSAIREN (National Institute of Neurological Disorders and Stroke and Association Internationale pour la Recherché et l'Enseignement en Neurosciences) criteria suggesting vascular dementia were excluded. Subjects were excluded also if a finding suggested the following possible nonneurodegenerative causes of cognitive decline: clinically significant abnormality on screening blood tests including vitamin $B_{12}$ level and thyroid function tests, a Geriatric Depression Score greater than 6 , a history of seizure disorder, a history of cranial radiation therapy, a history of mental retardation, a recent history of focal brain injury, focal neurologic deficits that developed simultaneously with cognitive complaints, or the presence of a systemic or medical illness that would confound the diagnosis of a degenerative dementia. The Institutional Review Board of the University of Michigan (IRBMED) approved this investigation, and all participants signed an IRBMED-approved written informed consent.

\section{Clinical Classification}

Clinical classification was performed as described previously (18). Clinical evaluations included history and neurologic examination, brain MR imaging, laboratory evaluation to exclude potential confounders, and a standardized neuropsychologic evaluation. The neuropsychologic evaluation administered to all subjects included the measures from the National Alzheimer Coordinating Center Unified Dataset, consisting of the Mini-Mental State Examination, Boston Naming Test, Digit Span Forwards and Backwards, Trail Making Test (parts A and B), Logical Memory and Logical Memory Delayed Recall, and Semantic Fluency (Animal Naming). Caregivers completed the Neuropsychiatric Inventory. Clinical, structural imaging, laboratory, and neuropsychologic data for each subject were abstracted into a standard form by 1 of the investigators. A panel of experienced clinicians masked to the PET neuroimaging reviewed these data and assigned a classification to each subject with reference to recommended clinical criteria for MCI and MCI subtypes (amnestic MCI [aMCI], multidomain MCI [mdMCI], and nonamnestic MCI [naMCI]) based on clinical and neuropsychologic criteria (19). If there was discrepancy between raters, consensus was reached by discussion.

Subjects' neurologic and neuropsychologic examinations were repeated in 2- to 4-y intervals from the initial consensus evaluation. Clinical classification was repeated using the same consensus procedure and the same raters, who were masked to the initial clinical consensus evaluations. Consensus assessment included evaluation of progression to dementia. Subjects with persistent MCI were classified as described above. Subjects converting to dementia were then classified as 1 of the 3 major forms of neurodegenerative dementia using standard criteria for AD, LBD, and FTD (20-22).

\section{PET Imaging}

Subjects underwent ${ }^{11} \mathrm{C}$-PiB and ${ }^{11} \mathrm{C}$-dihydotetrabenazine PET imaging on a Siemens ECAT $\mathrm{HR}^{+}$camera operated in 3-dimensional mode (septa retracted), and they were scanned within 2-3 wk of initial evaluation. The 2 radiotracer scans were usually performed on the same half day, with at least $2 \mathrm{~h}$ between scans to allow for physical decay of the first tracer before the second scan. ${ }^{11} \mathrm{C}-\mathrm{PiB}$ was administered as an intravenous bolus of $45 \%$ of the total mass dosage over $30 \mathrm{~s}$, followed by constant infusion of the remaining $55 \%$ of the dose over the 80-min study duration (23). ${ }^{11} \mathrm{C}$-PiB PET images were acquired as a dynamic series of 17 scan frames over a total of $80 \mathrm{~min}$ as follows: $4 \times 30 \mathrm{~s}, 3 \times 1 \mathrm{~min}, 2 \times 2.5 \mathrm{~min}$, $2 \times 5 \mathrm{~min}$, and $6 \times 10 \mathrm{~min}$. Parametric ${ }^{11} \mathrm{C}-\mathrm{PiB}$ distribution volume ratio (DVR) images were computed by averaging the last 4 scan frames (40-80 $\mathrm{min}$ ) normalized to the mean value in the cerebellar hemisphere cortical gray matter. $(+)-{ }^{11} \mathrm{C}$-dihydotetrabenazine was administered intravenously as a bolus containing $55 \%$ of the total mass dose over $30 \mathrm{~s}$, followed by continuous infusion of the remaining $45 \%$ of the dose over the 60 -min study duration (24). A dynamic series of PET images was acquired over $60 \mathrm{~min}: 4 \times 30 \mathrm{~s}, 3 \times 1 \mathrm{~min}$, $2 \times 2.5 \mathrm{~min}, 2 \times 5 \mathrm{~min}$, and $4 \times 10 \mathrm{~min}$. Parametric ${ }^{11} \mathrm{C}$-dihydotetrabenazine DVR images were computed by averaging the last 3 scan frames (30-60 $\mathrm{min}$ ) and normalized to the mean value in the occipital cerebral cortex. This bolus-plus-infusion approach leads to steady-state or equilibrium conditions, and the tissue concentration ratio yields DVR directly.

\section{PET Neuroimaging Classifications}

PET image classifications were made by an expert familiar with the biodistributions of the tracers in normal and relevant pathologic conditions. Following the procedure used in our prior study and the phase III study of ${ }^{123}$ I-FP-CIT SPECT for diagnosis of LBD, the assessments were designed to reproduce conditions that would likely be obtained if routine clinical use of these measures was implemented $(18,25)$. Parametric ${ }^{11} \mathrm{C}-\mathrm{PiB}$ and ${ }^{11} \mathrm{C}$-dihydotetrabenazine DVR image sets for each subject were stripped of identifiers and then classified without knowledge of clinical data. DVR images from all subjects were displayed with a DVR scale maximum of 3.0. Image-based classifications were assigned on the basis of combined ${ }^{11} \mathrm{C}$-PiB and ${ }^{11} \mathrm{C}$-dihydotetrabenazine results in each subject, providing both ligand-binding pattern and ligand-binding magnitude information. Subjects were classified as abnormal if visual assessment of ${ }^{11} \mathrm{C}$-dihydotetrabenazine DVR images indicated marked reduction of striatal ${ }^{11} \mathrm{C}$-dihydotetrabenazine binding in either hemisphere with visual assessment of the DVR images. Cerebral ${ }^{11} \mathrm{C}-\mathrm{PiB}$ retention was classified as abnormal when the frontal lobe cerebral cortical DVR exceeded subjacent frontal white matter DVR with visual inspection of the DVR images. The visual assessment of cortical ${ }^{11} \mathrm{C}-\mathrm{PiB}$ retention is known to exhibit accuracy comparable to quantitative analyses of ${ }^{11} \mathrm{C}-\mathrm{PiB}$ binding $(26,27)$. In our prior study, qualitative visual classification was as effective as classification based on regional parametric analysis (18). Subjects with markedly reduced striatal ${ }^{11} \mathrm{C}$-dihydotetrabenazine binding were classified as LBD. In our present series, some LBD subjects 
had ${ }^{11} \mathrm{C}$-PiB deposition, whereas others were ${ }^{11} \mathrm{C}$-PiB-negative. Individuals with normal ${ }^{11} \mathrm{C}$-dihydotetrabenazine binding and abnormally increased ${ }^{11} \mathrm{C}-\mathrm{PiB}$ deposition were classified as AD. Individuals with normal ${ }^{11} \mathrm{C}-\mathrm{PiB}$ and ${ }^{11} \mathrm{C}$-dihydotetrabenazine scan results were classified as FTD. Representative images of AD, LBD, and FTD classifications were published previously as Figure 1 in our prior study (18).

\section{Statistical Analyses}

Data analysis was performed with Stata (version 12; Stata Corp.). Demographics and other characteristics of the subjects were summarized as frequencies and percentages for categoric variables and as mean and SD for continuous variables. Differences between groups on demographic and neuropsychologic variables were assessed using $\chi^{2}$ tests for categoric variables, $t$ tests or 1-way ANOVA for normally distributed variables, and Mann-WhitneyWilcoxon or Kruskal-Wallis rank-sum tests for nonnormally distributed variables. $\kappa$-statistics were calculated to assess interassessment agreement.

\section{RESULTS}

We evaluated 27 subjects whom we were able to follow over a mean interval of approximately 3 y (Table 1). At study entry, 11 subjects were clinically classified as aMCI, 7 as mdMCI, and 9 as naMCI. The MCI classification subgroups were similar in terms of demographics, clinical features, and neuropsychologic features (Table 1). Two thirds of subjects converted to dementia (18/27) on follow-up consensus clinical assessments. For each MCI classification subgroup, approximately two-thirds converted to dementia (Table 2). With imaging-based classification, most subjects were classified as AD-like pathology (18/27), with minorities classified as LDB-like pathology (2/27) or FTD-like pathology $(7 / 27)$ (Table 2). Most of the subjects with an imaging abnormality (positive ${ }^{11} \mathrm{C}$-PiB PET or ${ }^{11} \mathrm{C}$-dihydotetrabenazine PET result) converted to dementia (16/20), whereas a minority of those without imaging abnormalities converted to dementia (2/7; Table 2; odds ratio, 10.0 [95\% confidence interval, 1.4-71.9]).

Although the numbers of subjects are relatively small, MCI classification subgroup status did not obviously correlate with image-based classification (Table 3). Among subjects, for example, categorized as aMCI, most (9/11) received imagebased classifications of AD but a minority (2/11) was categorized as FTD. Similarly, subjects classified as AD by image-based criteria were clinically classified as aMCI (9/18), mdMCI (6/18), and naMCI (3/18).

Using data from the 18 subjects who converted to dementia, we updated our prior analysis comparing PET imaging-based classification and expert clinical consensus classification of mildly demented subjects (Mini-Mental State Examination $>17$ ) (18). Adding this group of MCI subjects

TABLE 1

Demographic, Clinical, and Neuropsychologic Features of $\mathrm{MCl}$ Subjects

\begin{tabular}{|c|c|c|c|c|}
\hline Parameter & $\mathrm{aMCl}$ & mdMCl & naMCl & $P$ \\
\hline \multicolumn{5}{|l|}{ Demographic } \\
\hline Age $(y)$ & $74.5(8.1)$ & $70.3(9.7)$ & $73(9.8)$ & 0.41 \\
\hline Percentage female & $9(81.8 \%)$ & $3(42.9 \%)$ & $3(33.3 \%)$ & 0.07 \\
\hline White & $10(90.9 \%)$ & $6(85.7 \%)$ & $9(100 \%)$ & 0.536 \\
\hline Education (y) & $15.0(3.2)$ & $16.4(3.5)$ & $16.2(2.8)$ & 0.608 \\
\hline \multicolumn{5}{|l|}{ Clinical feature } \\
\hline Clinical Dementia Rating & & & & 0.434 \\
\hline 0 & $0(0 \%)$ & $0(0 \%)$ & $1(11.1 \%)$ & \\
\hline 0.5 & $8(72.7 \%)$ & $4(57.1 \%)$ & 7 (77.8\%) & \\
\hline 1 & $3(27.3 \%)$ & $3(42.9 \%)$ & $1(11.1 \%)$ & \\
\hline Mini-Mental State Examination & $25.5(2.8)$ & $25.9(1.1)$ & $27.2(2.4)$ & 0.292 \\
\hline Neuropsychiatric Inventory total score & $2.9(2.5)$ & $2.3(1.5)$ & $2.4(2.7)$ & 0.858 \\
\hline Neuropsychiatric Inventory severity & $4.6(4.4)$ & $3.4(2.6)$ & $3.7(4.9)$ & 0.755 \\
\hline Memory complaint & $11(100 \%)$ & $7(100 \%)$ & $7(77.8 \%)$ & 0.115 \\
\hline Behavior complaint & $0(0 \%)$ & $0(0 \%)$ & $2(22.2 \%)$ & 0.115 \\
\hline Parkinsonism & $0(0 \%)$ & $0(0 \%)$ & $2(22.2 \%)$ & 0.115 \\
\hline Hallucinations & $0(0 \%)$ & $0(0 \%)$ & $0(0 \%)$ & \\
\hline \multicolumn{5}{|l|}{ Neuropsychologic indices } \\
\hline Logical memory & $8.5(5)$ & $5.9(2.4)$ & $11.7(5.1)$ & 0.067 \\
\hline Digit Span Forward & $7.7(1.6)$ & $7.6(2)$ & $7(2.6)$ & 0.74 \\
\hline Digit Span Backward & $6.2(2.1)$ & $4.1(0.9)$ & $5.1(1.7)$ & 0.06 \\
\hline Animals & $15.6(4.3)$ & $13.7(5)$ & $14.7(4.4)$ & 0.647 \\
\hline \multicolumn{5}{|l|}{ Trail Making Test } \\
\hline Part A & $36.7(13.5)$ & $44.9(20.6)$ & $150.7(317.7)$ & 0.429 \\
\hline Part B & $192.5(269.3)$ & $183.4(106.4)$ & $373.7(362.4)$ & 0.147 \\
\hline Wechsler Adult Intelligence Scale & $40(11.1)$ & $32(11.7)$ & $33.2(7.8)$ & 0.425 \\
\hline Delayed Recall & $5(4.9)$ & $4(2.3)$ & $10.9(5)$ & 0.018 \\
\hline Boston Naming Test & $26.4(2.7)$ & $22.3(8.4)$ & $26.2(3.5)$ & 0.625 \\
\hline Duration of follow-up (mo) & $32.5(14.3)$ & $37.3(14.9)$ & $40.0(12.0)$ & 0.399 \\
\hline
\end{tabular}

Data in parentheses are percentages (when followed by \%) or are SDs. 
TABLE 2

Results of Subject Follow-up Based on Either Initial Clinical or Initial Image-Based Classifications

\begin{tabular}{lccc}
\hline Classification & Total & Did not convert to dementia & Did convert to dementia \\
\hline Baseline clinical & 11 & 4 & 7 (7 AD) \\
aMCl & 7 & 2 & 5 (4 AD and 1 FTD) \\
mdMCl & 9 & 3 & 4 LBD, 1 AD, and 1 FTD) \\
naMCl & 18 & 4 & $14(11$ AD, 2 LBD, and 1 FTD) \\
Baseline image-based & 2 & 0 & $2(1$ LBD and 1 FTD) \\
AD & 7 & 5 & $2(1$ AD and 1 LBD) \\
LBD & & & \\
FTD* & &
\end{tabular}

converting to dementia to our prior cohort of 75 mildly demented subjects provides a total of 93 subjects for comparison (Table 4). Updated analysis results are similar to prior results. The overall Cohen's $\kappa$ was 0.41 (95\% confidence interval, 0.26-0.55), and the performance characteristics of expert clinical consensus image-based classification were similar to our previously reported results (Table 5). As with our prior analysis, the greatest source of discrepancy between image-based and clinical classifications was in subjects classified clinically as FTD but whose ${ }^{11} \mathrm{C}-\mathrm{PiB}$ PET imaging revealed significant cerebral $\mathrm{A} \beta$ amyloid deposition (Table 6).

\section{DISCUSSION}

A general problem with disease-modifying clinical trials in demented subjects is that pathology may be so advanced as to substantially reduce or preclude detectable therapeutic effects. MCI subject populations are likely less susceptible to this problem. A further virtue of targeting MCI subjects is that a trial endpoint of conversion to dementia may allow employment of survival analysis methods, increasing trial statistical power, an approach precluded in trial designs with demented subjects. With high risk for conversion to dementia and probable lower intensity of pathology, MCI is an attractive target population for disease-modifying trials. MCI populations, however, include both subjects with underlying progressive illnesses and subjects who will not progress or will actually improve. MCI secondary to dementing disorders, such as early dementia, is a heterogeneous population with several underlying pathologies. Identification of MCI subjects most likely to progress to dementia would increase the statistical power of trials to identify clinically relevant effects. Accurate, pathologically based classification of MCI subjects would facilitate enrollment of more homogeneous subject populations and also increase statistical power in disease-modifying trials.

Amyloid imaging and nigrostriatal dopamine terminal imaging are well validated by postmortem studies as biomarkers for cerebral $\mathrm{A} \beta$ amyloid deposition and LBD $(16,17,28,29)$. In our dataset, abnormal ${ }^{11} \mathrm{C}$-dihydotetrabenazine or ${ }^{11} \mathrm{C}$-PiB PET results strongly predicted progression to dementia, which is consistent with considerable data from prior amyloid imaging studies of MCI subjects (30-32). Amyloid imaging does not, however, differentiate AD from LDB because many LDB subjects exhibit high amyloid burden (33-35). In addition, LBD subjects without high amyloid burdens will not be identified by amyloid imaging. Our combined molecular imaging approach likely identifies MCI subjects at high risk for progression and may allow relatively specific identification of MCI subjects likely to progress to $\mathrm{AD}$ and $\operatorname{LBD}(18,36,37)$.

An alternative approach to increasing the likelihood of identifying MCI subjects at high risk for progression to dementia is to use clinically defined MCI subclassifications as markers for increased chance for progression to dementia. aMCI, for example, is proposed as a particularly strong risk factor for AD (38). This approach, however, will still likely result in heterogeneous subject populations. Although our number of MCI subjects is modest, the lack of qualitative association of any MCI subtype with image-based classification is consistent with the heterogeneity of MCI and MCI subtypes. Of the 11 subjects in our study classified clinically as aMCI, 9 were classified by imaging criteria as AD (high ${ }^{11} \mathrm{C}$-PiB binding and normal ${ }^{11} \mathrm{C}$-dihydotetrabenazine binding) with $2(18 \%)$ classified as FTD (normal ${ }^{11} \mathrm{C}-\mathrm{PiB}$ and ${ }^{11} \mathrm{C}$-dihydotetrabenazine binding). This result is similar to findings in a phase II trial of bapinuzemab in $\mathrm{AD}$, in which $15 \%$ of subjects enrolled with clinically defined mild AD were found subsequently to have normal ${ }^{11} \mathrm{C}-\mathrm{PiB}$ PET results

TABLE 3

$\mathrm{MCl}$ Classifications

\begin{tabular}{lcccc}
\hline Classification & AD $^{*}$ & LBD & FTD & Total \\
\hline aMCl $^{\dagger}$ & 9 & 0 & 2 & 11 \\
mdMCl & 6 & 0 & 1 & 7 \\
naMCl & 3 & 2 & 4 & 9 \\
Total & 18 & 2 & 7 & 27 \\
& \\
*Image-based classification of MCl subjects (AD, LBD, FTD). \\
†Initial expert clinical consensus classification of MCl (aMCl, \\
mdMCl, naMCl).
\end{tabular}


TABLE 4

Demographic, Clinical, and Neuropsychologic Features of $\mathrm{MCl}$ and Demented Subjects

\begin{tabular}{|c|c|c|c|c|}
\hline Parameter & $\mathrm{AD}(n=48)$ & $\operatorname{LBD}(n=18)$ & FTD $(n=27)$ & $P$ \\
\hline \multicolumn{5}{|l|}{ Demographic } \\
\hline Age (y) & $71.4(8.8)$ & $71.6(7.4)$ & $66.1(8.5)$ & 0.031 \\
\hline Percentage female & $22(45.8 \%)$ & $6(33.3 \%)$ & $12(44.4 \%)$ & 0.648 \\
\hline White & $45(93.8 \%)$ & $16(88.9 \%)$ & $27(100 \%)$ & 0.250 \\
\hline Education (y) & $15(3.1)$ & $14.8(2.9)$ & $15.5(3.4)$ & 0.777 \\
\hline \multicolumn{5}{|l|}{ Clinical feature } \\
\hline Clinical Dementia Rating & & & & 0.497 \\
\hline 0 & $0(0 \%)$ & $0(0 \%)$ & $1(3.7 \%)$ & \\
\hline 0.5 & $18(37.5 \%)$ & $9(50 \%)$ & $10(37 \%)$ & \\
\hline 1 & $30(62.5 \%)$ & $9(50 \%)$ & $16(59.3 \%)$ & \\
\hline Mini-Mental State Examination & $23.4(3.1)$ & $23.1(4.4)$ & $22.3(3.9)$ & 0.466 \\
\hline Neuropsychiatric Inventory total score & $2.8(2.3)$ & $2.6(2.5)$ & $2.7(2.2)$ & 0.867 \\
\hline Neuropsychiatric Inventory severity & $4.1(4)$ & $4.2(5)$ & $4.4(4.3)$ & 0.840 \\
\hline Memory complaint & $43(89.6 \%)$ & $16(88.9 \%)$ & $21(77.8 \%)$ & 0.340 \\
\hline Behavior complaint & $5(10.4 \%)$ & $4(22.2 \%)$ & $9(33.3 \%)$ & 0.052 \\
\hline Parkinsonism & $3(6.2 \%)$ & $13(72.2 \%)$ & $1(3.7 \%)$ & 0.000 \\
\hline Hallucinations & $0(0 \%)$ & $8(44.4 \%)$ & $1(3.7 \%)$ & 0.000 \\
\hline \multicolumn{5}{|l|}{ Neuropsychologic indices } \\
\hline Logical memory & $5.8(3.9)$ & $8.9(4.6)$ & $7(5)$ & 0.046 \\
\hline Digit Span Forward & $7.4(1.8)$ & $6.8(2.9)$ & $5.7(2.5)$ & 0.023 \\
\hline Digit Span Backward & $4.7(1.6)$ & $4.5(2)$ & $3.7(1.7)$ & 0.107 \\
\hline Animals & $12.5(4.7)$ & $11.3(4.5)$ & $11.7(4.7)$ & 0.486 \\
\hline \multicolumn{5}{|l|}{ Trail Making Test } \\
\hline Part A & $56.4(33.7)$ & $84.6(42.6)$ & 172.1 (298.9) & 0.016 \\
\hline Part B & $431.5(374.6)$ & $428.6(316.2)$ & $576.6(387.8)$ & 0.223 \\
\hline Wechsler Adult Intelligence Scale & $31.5(17.5)$ & $24.3(19.3)$ & $30.4(25.7)$ & 0.038 \\
\hline Delayed Recall & $3.6(3.5)$ & $8(4.7)$ & $9.6(17.7)$ & 0.001 \\
\hline Boston Naming Test & $23.2(5)$ & $24.1(4.6)$ & $23.2(5.8)$ & 0.822 \\
\hline
\end{tabular}

Data in parentheses are percentages (when followed by \%) or are SDs.

(39). Similarly, all forms of clinically defined MCI were represented among our subjects classified as AD by image-based criteria (Table 3).

One drawback of this approach is that both the clinical and the molecular imaging-based classification schemes presume exclusive categories. Combinations of pathology, notably comorbid vascular and neurodegenerative pathologies, are common in large autopsy series of demented subjects. Although conventional, this classification approach inevitably obscures some of the complexities of overlapping pathologies contributing to the clinical features of dementing illnesses. Some FTD cases, for example, notably those associated with $\tau$ mutations, exhibit significant parkinsonism and loss of nigrostriatal neurons. In addition, our molecular imaging-based FTD classification is a negative approach, based on absence of ${ }^{11} \mathrm{C}$-dihydotetrabenazine PET or ${ }^{11} \mathrm{C}$-PiB PET abnormalities. In the case of demented individuals, for example, this approach could lead to individuals with vascular dementia being classified as FTDs. We attempted to mitigate this problem by excluding subjects with higher Hachinski scores, but misclassification of vascular cognitive impairment as FTD remains a possibility with this approach. Similarly, this negative approach to FTD classification would also lead to subjects without dementia-related pathologies being classified as FTD. This fact highlights the importance of developing new ligands specific for FTD-related pathologies, such as $\tau$-protein or TAR-DNA-binding protein-43 deposition, that would serve as positive biomarkers.

Another limitation of our study is the relatively small sample size. Only 2 subjects converting from MCI to

TABLE 5

Performance of Clinical Diagnosis* Versus Image-Based Classification in 93 Subjects

\begin{tabular}{ccccccc}
\hline Classification & Image-based & Sensitivity & Specificity & Positive predictive value & Negative predictive value & $\kappa$ \\
\hline Clinical & AD & 0.67 & 0.78 & 0.85 & 0.55 & 0.41 \\
& FTD & 0.6 & 0.77 & 0.33 & 0.91 & 0.28 \\
& LBD & 0.65 & 0.91 & 0.61 & 0.92 & 0.54
\end{tabular}

*Expert clinical consensus diagnosis. Analysis assumes that image-based classification is gold standard. 
TABLE 6

Comparison of Image-Based and Clinical Consensus Classifications

\begin{tabular}{lcccc}
\hline & \multicolumn{3}{c}{ Image-based } \\
\cline { 2 - 3 } Classification & AD & LBD & FTD & Total \\
\hline Clinical & & & & \\
AD & $41^{*}$ & 4 & 3 & 48 \\
LBD & 4 & $11^{\star}$ & 3 & 18 \\
\hline FTD & 16 & 2 & $9^{*}$ & 27 \\
Total & 61 & 17 & 15 & 93 \\
\hline
\end{tabular}

*Subjects with concordant clinical classifications. Largest discrepant group is subjects classified as FTD but meeting image-based criteria for $A D$ - that is, elevated ${ }^{11} \mathrm{C}-\mathrm{PiB}$ binding.

dementia exhibited abnormal striatal ${ }^{11} \mathrm{C}$-dihydotetrabenazine binding with initial PET imaging. Most of the MCI-todementia convertors exhibited abnormal ${ }^{11} \mathrm{C}$-PiB PET imaging. Demonstrating that this double-PET-scan approach is superior to single imaging with ${ }^{11} \mathrm{C}-\mathrm{PiB} \mathrm{PET}$ or another imaging modality will require a larger prospective study. One potentially interesting result was that 3 of the 4 subjects classified as converting to LBD with the final consensus clinical evaluation were not classified as LBD on initial imaging. The most likely explanation for discrepant imaging and clinical classifications is imprecision of clinical classification, but another intriguing possibility exists. We previously reported apparently rapid loss of striatal ${ }^{11} \mathrm{C}$-dihydotetrabenazine binding in a subject with rapid-eye-movement sleep behavior disorder who converted to LBD (40). This prior case experience raises the possibility of apparently rapid declines in striatal dopaminergic innervation in LBD during phases when subjects convert from MCI or other potential precursor states to overt dementia. This finding raises the interesting possibility that dopamine terminal PET or SPECT imaging, although generally thought to be useful in establishing a diagnosis of LBD in individuals in established dementia, may be insensitive in precursor states like MCI. Larger prospective studies are required to address this question.

We previously applied this combined-tracer PET imaging approach to subjects with early, mild dementia. Concordance with expert clinical diagnosis was only moderate, consistent with the results of long-term pathologic follow-up of dementias (41). We extended our prior analysis of concordance between molecular imaging-based and clinical classifications of early dementia by adding the results of final expert clinical consensus and image-based classifications of the $18 \mathrm{MCI}$ subjects who converted to dementia to our prior dataset. With this expanded dataset (93 subjects), we continue to see only moderate concordance $(\kappa=0.41)$ between expert clinical consensus and molecular imaging-based classifications. We suspect that discrepancies between imaging and clinical classifications reflect imprecision of clinical classifications, but pathologic correlation is necessary to assess this hypothesis definitively.
This combined-tracer approach may be useful for selecting MCI subjects for inclusion in disease-modifying therapy trials both by selecting populations at high risk for conversion to dementia and by selecting a more homogeneous subject population on the basis of underlying pathologic-neurochemical abnormalities. Alternatively, this approach may be useful in defining more accessible biomarkers in MCI subjects. Other measures, such as ${ }^{18} \mathrm{~F}$-FDG PET and structural MR imaging, have been explored as biomarkers to predict progression from MCI to dementia and for a more accurate classification of MCI or dementias. Significant results are reported with these imaging modalities. Determining the optimal approach for assessing prognosis and classification will require larger prospective studies with pathologic correlation as the ultimate gold standard.

\section{CONCLUSION}

Combined PET molecular imaging of cerebral amyloid burden and striatal dopamine terminal integrity may be useful in identifying subjects at high risk for progression to dementia and in defining neurochemically differentiated subsets of MCI subjects.

\section{DISCLOSURE}

The costs of publication of this article were defrayed in part by the payment of page charges. Therefore, and solely to indicate this fact, this article is hereby marked "advertisement" in accordance with 18 USC section 1734. Roger L. Albin accessed all relevant data for this study and takes responsibility for integrity of data and analysis. The sponsors played no role in data acquisition, analysis, interpretation or manuscript preparation. This study was supported in part by the following: PHS P01 NS15655 and P30 AG08671 and a gift from an anonymous donor.

The authors make the following disclosures:

Roger L. Albin receives research support from NIH and the Department of Veteran's Affairs. He serves on the DSMB of the QE3 trial and is a member of the Scientific Advisory Board of the Hereditary Disease Foundation and serves on the Editorial Boards of Neurology, Experimental Neurology, and Neurobiology of Disease.

James F. Burke receives support from a VA advanced fellowship and the Robert Wood Johnson Clinical Scholars Program.

Robert A. Koeppe receives research support from NIH. Dr. Koeppe has served as a consultant for Johnson \& Johnson and Merck and serves on the board of the International Society of Cerebral Blood Flow and Metabolism.

Bruno Giordani receives research support from NIH.

Sid Gilman is chair of DSMB for Bapineuzumab phase III study of AD, Elan \& Janssen AI; chair of DSMB for AAB003 Phase I, Pfizer; and chair of DSMB for ELND005 Phase III, Elan. He receives research support from NINDS (NIH) and is on the scientific advisory boards of Longitude Capital and TPP. 
Kirk A. Frey receives research support from USPHS NIH and GE Healthcare, AVID Radiopharma; he serves as consultant to MiMvista, GE Healthcare, Avid Radiopharma (Eli Lilly), and Bayer-Schering Pharma; and has equity (stock) ownership in Bristol-Myers Squibb, General Electric, Johnson \& Johnson, Merck, and Novo Nordisk.

No other potential conflict of interest relevant to this article was reported.

\section{ACKNOWLEDGMENTS}

We thank the staff of the PET center for their assistance. We thank the reviewers for useful criticisms and the $J N M$ staff for careful editing.

\section{REFERENCES}

1. Petersen RC, Smith GE, Waring SC, Ivnik RJ, Tangalos EG, Kokmen E. Mild cognitive impairment: clinical characteristics and outcome. Arch Neurol. 1999; 56:303-308

2. Markesbery WR, Schmitt FA, Kryscio RJ, Davis DG, Smith CD, Wekstein DR. Neuropathologic substrate of mild cognitive impairment. Arch Neurol. 2006; 63:38-46.

3. Bennett DA, Schneider JA, Bienias JL, Evans DA, Wilson RS. Mild cognitive impairment is related to Alzheimer disease pathology and cerebral infarctions. Neurology. 2005;64:834-841.

4. Guillozet AL, Weintraub S, Mash DC, Mesulam MM. Neurofibrillary tangles, amyloid, and memory in aging and mild cognitive impairment. Arch Neurol. 2003;60:729-736.

5. Petersen RC, Parisi JE, Dickson DW, et al. Neuropathologic features of amnestic mild cognitive impairment. Arch Neurol. 2006;63:665-672.

6. Jicha GA, Parisi JE, Dickson DW, et al. Neuropathological outcome of mild cognitive impairment following progression to clinical dementia. Arch Neurol. 2006;63:674-681.

7. Molano J, Boeve B, Ferman T, et al. Mild cognitive impairment associated with limbic and neocortical Lewy body disease: a clinicopathological study. Brain. 2010;133:540-556.

8. Loewenstein DA, Acevedo A, Small BJ, Agron J, Crocco E, Duara R. Stability of different subtypes of mild cognitive impairment among the elderly over a 2- to 3-year follow-up period. Dement Geriatr Cogn Disord. 2009;27:418423.

9. Palmer K, Bäckman L, Winblad B, Fratiglioni L. Mild cognitive impairment in the general population: occurrence and progression to Alzheimer disease. Am J Geriatr Psychiatry. 2008;16:603-611.

10. Mitchell AJ, Shiri-Feshki M. Rate of progression of mild cognitive impairment to dementia: meta-analysis of 41 robust inception cohort studies. Acta Psychiatr Scand. 2009;119:252-265.

11. Farias ST, Mungas D, Reed BR, Harvey D, DeCarli C. Progression of mild cognitive impairment to dementia in clinic- vs community-based cohorts. Arch Neurol. 2009;66:1151-1157.

12. Manly JJ, Tang MX, Schupf N, Stern Y, Vonsattel JP, Mayeux R. Frequency and course of mild cognitive impairment in a multiethnic community. Ann Neurol. 2008;63:494-506.

13. Klunk WE, Engler H, Nordberg A, et al. Imaging brain amyloid in Alzheimer's disease with Pittsburgh Compound-B. Ann Neurol. 2004;55:306-319.

14. Koeppe RA, Gilman S, Junck L, Wernette K, Frey KA. Differentiating Alzheimer's disease from dementia with Lewy bodies and Parkinson's disease with $(+)-\left[{ }^{11} \mathrm{C}\right]$ dihydrotetrabenazine positron emission tomography. Alzheimers Dement. 2008;4 (1, suppl 1):S67-S76.

15. Clark CM, Schneider JA, Bedell BJ, et al. Use of florbetapir-PET for imaging beta-amyloid pathology. JAMA. 2011;305:275-283.

16. Suzuki M, Desmond TJ, Albin RL, Frey KA. Striatal monoaminergic terminals in Lewy body and Alzheimer's dementias. Ann Neurol. 2002;51:767-771.
17. Piggott MA, Marshall EF, Thomas N, et al. Striatal dopaminergic markers in dementia with Lewy bodies, Alzheimer's and Parkinson's diseases: rostrocaudal distribution. Brain. 1999;122:1449-1468.

18. Burke JF, Albin RL, Koeppe RA, et al. Assessment of mild dementia with amyloid and dopamine terminal positron emission tomography. Brain. 2011; 134:1647-1657.

19. Winblad B, Palmer K, Kivipelto M, et al. Mild cognitive impairment: beyond controversies, towards a consensus: report of the International Working Group on Mild Cognitive Impairment. J Intern Med. 2004;256:240-246.

20. McKhann G, Drachman D, Folstein M, et al. Clinical diagnosis of Alzheimer's disease: report of the NINCDS-ADRDA work group under the auspices of Department of Health and Human Services task force on Alzheimer's disease. Neurology. 1984;34:939-944.

21. McKhann GM, Albert MS, Grossman M, et al. Clinical and pathological diagnosis of frontotemporal dementia. Arch Neurol. 2001;58:1803-1809.

22. McKeith IG, Dickson DW, Lowe J, et al. Diagnosis and management of dementia with Lewy bodies: third report of the DLB consortium. Neurology. 2005;65: 1863-1872.

23. Koeppe RA, Frey KA. Equilibrium analysis of $\left[{ }^{11} \mathrm{C}\right] \mathrm{PIB}$ studies. Neuroimage. 2008;41:T30.

24. Bohnen NI, Albin RL, Koeppe RA, et al. Positron emission tomography of monoaminergic vesicular binding in aging and Parkinson disease. J Cereb Blood Flow Metab. 2006;26:1198-1212.

25. McKeith I, O'Brien J, Walker Z, et al. Sensitivity and specificity of dopamine transporter imaging with ${ }^{123}$ I-FP-CIT SPECT in dementia with Lewy bodies: a phase III, multicentre study. Lancet Neurol. 2007;6:305-313.

26. Ng S, Villemagne VL, Berlangieri S, et al. Visual assessment versus quantitative assessment of ${ }^{11} \mathrm{C}$-PIB PET and ${ }^{18} \mathrm{~F}$-FDG PET for detection of Alzheimer's disease. J Nucl Med. 2007;48:547-552.

27. Suotunen T, Hirvonen J, Immonen-Räihä P, et al. Visual assessment of [ $\left.{ }^{11} \mathrm{C}\right] \mathrm{PIB}$ PET in patients with cognitive impairment. Eur J Nucl Med Mol Imaging. 2010;37:1141-1147.

28. Fodero-Tavoletti MT, Smith DP, McLean CA, et al. In vitro characterization of Pittsburgh compound-B binding to Lewy bodies. J Neurosci. 2007;27:10365-10371.

29. Klunk WE, Wang Y, Huang GF, et al. The binding of 2-(4-methylaminophenyl) benzothiazole to postmortem brain homogenates is dominated by the amyloid component. J Neurosci. 2003;23:2086-2092.

30. Wolk DA, Price JC, Saxton JA, et al. Amyloid imaging in mild cognitive impairment subtypes. Ann Neurol. 2009;65:557-568.

31. Okello A, Koivunen J, Edison P, et al. Conversion of amyloid positive and negative MCI to AD over 3 years: an ${ }^{11}$ C-PIB PET study. Neurology. 2009;73:754-760.

32. Villemagne VL, Pike KE, Chételat G, et al. Longitudinal assessment of A $\beta$ and cognition in aging and Alzheimer disease. Ann Neurol. 2011;69:181-192.

33. Gomperts SN, Rentz DM, Moran E, et al. Imaging amyloid deposition in Lewy body diseases. Neurology. 2008;71:903-910.

34. Edison P, Rowe CC, Rinne JO, et al. Amyloid load in Parkinson's disease dementia and Lewy body dementia measured with $\left[{ }^{11} \mathrm{C}\right] \mathrm{PIB}$ positron emission tomography. J Neurol Neurosurg Psychiatry. 2008;79:1331-1338.

35. Foster ER, Campbell MC, Burack MA, et al. Amyloid imaging of Lewy bodyassociated disorders. Mov Disord. 2010;25:2516-2523.

36. Villemagne VL, Okamura N, Pejoska S, et al. Differential diagnosis in Alzheimer's disease and dementia with Lewy bodies via VMAT2 and amyloid imaging. Neurodegener Dis. 2012;10:161-165.

37. Villemagne VL, Okamura N, Pejoska S, et al. In vivo assessment of vesicular monoamine transporter type 2 in dementia with Lewy bodies and Alzheimer disease. Arch Neurol. 2011;68:905-912.

38. Petersen RC, Thomas RG, Grundman M, et al. Vitamin E and donepezil for treatment of mild cognitive impairment. N Engl J Med. 2005;352:2379-2388.

39. Rinne JO, Brooks DJ, Rossor MN, et al. ${ }^{11} \mathrm{C}-\mathrm{PiB}$ PET assessment of change in fibrillar amyloid-beta load in patients with Alzheimer's disease treated with bapineuzumab: a phase 2, double-blind, placebo-controlled, ascending-dose study. Lancet Neurol. 2010;9:363-372.

40. Albin R, Koeppe R. Rapid loss of striatal VMAT2 binding associated with onset of Lewy body dementia. Mov Disord. 2006;21:287-288.

41. Knopman DS, DeKosky ST, Cummings JL, et al. Practice parameter: diagnosis of dementia (an evidence-based review). Report of the Quality Standards Subcommittee of the American Academy of Neurology. Neurology. 2001;56:1143-1153. 